\title{
Actitudes sexuales de acuerdo con el nivel de severidad del trauma craneoencefálico
}

\section{Sexual attitudes according to the level of severity of the cranioencephalic trauma}

Dubis Marcela Rincón Barreto", María Rocío Acosta Barreto", Diana Milena Fajardo Tejada”*

Universidad Católica Luis Amigó

Universidad de San Buenaventura

Recibido: 30 de enero de 2020-Aceptado: 9 de febrero de 2021-Publicado: 1 de enero de 2022

Forma de citar este artículo en APA:

Rincón Barreto, D. M., Acosta-Barreto, M. R., \& Fajardo-Tejada, D. M. (2022). Actitudes sexuales de acuerdo con el nivel de severidad del trauma craneoencefálico. Revista Colombiana de Ciencias Sociales, 13(1), 22-42. https://doi.org/10.21501/22161201.3527

\section{Resumen}

El objetivo de esta investigación fue analizar la actitud hacia la sexualidad en pacientes con antecedentes de trauma craneoencefálico (TCE), de acuerdo al nivel de severidad presentado. Método: se llevó a cabo un estudio de tipo descriptivo en una muestra de 126 personas, con edades comprendidas entre los 18 y 49 años, con antecedente de trauma craneoencefálico leve, moderado y severo, a quienes se les aplicó la Escala de Actitudes hacia la Sexualidad Ampliada (ATSS) y una encuesta de actitudes hacia la sexualidad. Resultados: se encontró que la mayoría de los participantes manifestaron haber sufrido cambios en su sexualidad tras el TCE, caracterizados por la disminución de la frecuencia de las relaciones y el deseo sexual,

· Magíster en Neuropsicología Clínica. Universidad de San Buenaventura, Bogotá. Docente del programa de Psicología, coordinadora de la Maestría en Neuropsicopedagogía, Universidad Católica Luis Amigó. Integrante del grupo de investigación Farmacodependencia y otras Adicciones, Medellín, Colombia. Contacto: dubis.rinconba@amigo.edu.co, https://scholar.google.es/ citations?user=eHaulOIAAAAJ\&hl=es ORCID: https://orcid.org/0000-0002-8322-889X

* Ph.D en Psicología. Universidad de San Buenaventura Bogotá. Integrante del grupo de investigación Avances en Psicología. Bogotá, Colombia. Contacto: macosta@usbbog.edu.co https:/l scholar.google.com/citations?user=ct8-WLcAAAAJ\&hl=es ORCID: https://orcid.org/0000-0002-9629-495X

-"- Magíster en Neuropsicología Clínica. Universidad de San Buenaventura, Bogotá. Contacto: dianafajardo10@gmail.com, ORCID: https://orcid.org/0000-0002-7538-2838 
sin embargo, evidencian una actitud positiva frente a la sexualidad. Conclusiones: si bien la sexualidad es considerada importante por los participantes del estudio, no se evidenció una conducta tendiente a la erotofilia. No se encontraron diferencias significativas respecto al nivel de severidad del trauma craneoencefálico.

\section{Palabras clave}

Trauma craneoencefálico; TCE; Actitudes sexuales; Sexualidad; Rehabilitación; Evaluación.

\section{Abstract}

The objective of this research was to analyze the attitude towards sexuality in patients with a history of cranioencephalic trauma (TCE), according to the level of severity presented. Method: a descriptive study was carried out in a sample of 126 people, aged between 18 and 49 years, with a history of mild, moderate, and severe head trauma, to whom the Scale of Attitudes towards Extended Sexuality -ATSS and a Survey of Attitudes towards Sexuality were applied. Results: it was found that most of the participants reported having suffered changes in their sexuality after TCE, characterized by a decrease in the frequency of relationships and sexual desire, however, they show a positive attitude towards sexuality. Conclusions: although sexuality is considered important by the study participants, there was no evidence of behavior tending to erotophilia. No significant differences were found regarding the level of severity of cranioencephalic trauma.

\section{Keywords}

Traumatic brain injury; ECT; Sexual attitudes; Sexuality; Rehabilitation; Evaluation. 


\section{Introducción}

El Trauma Craneoencefálico (TCE) se define como una lesión producida por un golpe o un trauma de carácter violento en el cerebro una vez se produce un choque con las diferentes paredes internas del cráneo, y que normalmente implica pérdida de conciencia. El TCE puede clasificarse atendiendo al mecanismo de acción (abierto o cerrado), según la localización del daño (frontal, occipital, etc.) y de acuerdo con el grado de severidad (leve, moderado, severo) (González, 2012).

La severidad del trauma puede establecerse teniendo en cuenta la duración de la pérdida de la conciencia, el tiempo de la amnesia postraumática, una fractura de cráneo, la presencia de hematomas intracerebrales o extracerebrales, entre otros asuntos (MacNeill \& Wedding, 2008), evaluados mediante la Escala de Coma de Glasgow (ECG) - una de las medidas más consistentes empleadas hoy en día (Silver et al., 2005; García-Molina et al., 2008; Stein et al., 2017)-, la cual valora separadamente las respuestas motoras, las respuestas verbales y la apertura de ojos, siendo un indicador para estandarizar la severidad del daño cerebral en la evaluación temprana, por lo que su uso típicamente consiste en la observación de las respuestas del paciente. Por ello, es posible identificar la gravedad del TCE mediante la suma de las puntuaciones obtenidas en cada uno de los apartados (respuestas motoras, respuestas verbales y apertura de ojos); un TCE severo es aquel con una ECG de 0 a 8 puntos, un TCE moderado comprendido entre 9 y 13 , y un TCE leve con una ECG comprendida entre 14 y 15.

Los TCE leves son aquellas lesiones cerebrales en las que el paciente, dentro de las primeras 48 horas a partir del impacto, sufre una pérdida de conciencia menor a 5 minutos y presenta una amnesia postraumática breve (Rodríguez et al., 2000; Barcena-Orbe et al., 2006). Según Varela et al. (2005), las personas con trauma craneoencefálico leve (TCEL) hacen parte de un grupo heterogéneo de pacientes que han presentado pérdida transitoria de conciencia, memoria o visión posterior al trauma, manteniendo puntajes en la Escala de Coma de Glasgow superiores a 13, sin degradación en las 48 horas sucesivas; en este grupo, aproximadamente el $75 \%$ de los pacientes son atendidos en un centro hospitalario.

Respecto a los TCE moderados, son de frecuente ocurrencia y generalmente presentan un alto porcentaje de lesiones intracraneales $(27 \%-32 \%)$ y una elevada necesidad de intervención neuroquirúrgica $(7,5 \%)$; la lesión que suele ser común en pacientes con este tipo de trauma es el hematoma subgaleal, seguido por el edema cerebral difuso, el edema cerebral focal, la hemorragia subaracnoidea sin apertura a sistema ventricular y la contusión hemorrágica por trauma directo (Mendoza et al., 2003). 
En cuanto al TCE severo, la mortalidad se aproxima al $50 \%$, cuyas causas frecuentes son los accidentes de tránsito y las caídas. Según la Organización Mundial de la Salud (OMS, 2015), 10 millones de personas en Estados Unidos de América con edades entre los 25 y 35 años sufren TCE anualmente y un $20 \%$ de los mismos presenta lesión cerebral grave a causa de colisiones vehiculares. La tasa de mortalidad del TCE severo es de aproximadamente $35 \%$ y la recuperación funcional es solo de entre $40 \%$ y $50 \%$. Generalmente, los TCE severos están asociados a lesiones de tipo primario o daños directos causados por el impacto del trauma o por mecanismos de aceleración-desaceleración y se pueden presentar como una contusión cortical, laceración cerebral, fractura de cráneo, lesión axonal, contusión del tallo o desgarro dural o venoso (Guzmán, 2008). Las contusiones más comunes se observan en la región orbital de los lóbulos frontales, la superficie lateral e inferior de los lóbulos temporales y el córtex por encima de la cisura de Silvio (Ariza-González et al., 2004).

Dentro de los aspectos neuropsicológicos del TCE suelen coexistir lesiones focales y difusas. El daño cerebral que presentan los TCE moderados y graves consiste en un patrón de daño no específico y generalizado, pero con gran afectación de los lóbulos frontal y temporal (Bigler, 2001; Fontaine et al., 1999), lo que implica la alteración de funciones cognitivas como la atención, la memoria, las funciones frontales, la emoción y la conducta. Es así que los cambios emocionales que tienen lugar como resultado del trauma cerebral incluyen la exageración o desaparición de la experiencia y la respuesta emocional. Tras un TCE, la ansiedad y la depresión son los trastornos emocionales más comunes, así como los cambios en la personalidad, que pueden ir desde una leve exacerbación de los rasgos previos hasta un cambio radical de la personalidad premórbida, principal causa de queja y de motivo de consulta por parte de los familiares (Gálvez-González et al., 2009; Castaño et al., 2012).

Los cambios más frecuentes son la apatía y la pérdida de interés por el entorno, aunque en otros casos se evidencia irritabilidad, impulsividad, desinhibición, euforia, labilidad emocional, puerilidad, suspicacia o intolerancia a la frustración, afectando las relaciones interpersonales. Frecuentemente, también se observa pérdida de la conciencia del propio déficit o una falta de habilidad para percibir los cambios en las funciones cognitivas o en la conducta (Guzmán, 2008; García-Molina et al., 2010; Spencer et al., 2019).

De igual manera, Arango-Lasprilla et al. (2007) afirman que la lesión cerebral puede afectar directa o indirectamente en aspectos como la sexualidad y la función sexual. Generalmente, los daños en el lóbulo frontal desencadenan conductas sexuales inapropiadas, falta de control de los impulsos sexuales, desinhibición, euforia, falta de tacto, o cambios en el sistema de activación sexual, así como disminución del deseo y de la excitación genital, pérdida de espontaneidad del individuo y falta de vitalidad; las lesiones en el hipotálamo basal pueden conducir a la pérdida total e irreversible del deseo sexual (Creuter et al., 2009; Albadan-Amaya et al., 2013). 
La sexualidad, que forma parte de la personalidad del individuo, se manifiesta progresivamente a lo largo de la vida y se concibe no solo como una necesidad fisiológica, sino también como una capacidad de comunicar, expresar, dar y obtener placer. En este proceso interactúan factores biológicos, psicológicos, sociales, económicos, políticos, culturales, éticos, legales, históricos, religiosos y espirituales, configurando así las actitudes hacia la sexualidad o la tendencia psicológica a evaluar favorable o desfavorablemente una conducta sexual (Bautista-Hernández, 2008; Castro et al., 2004).

Aquellos individuos que manifiestan actitudes sexuales positivas suelen gozar de una mayor satisfacción sexual (Ortega et al., 2005), e incluso demostrar más habilidades en la prevención de enfermedades de transmisión sexual, contrario a lo que se podría pensar de las personas con actitudes sexuales negativas. En general, las actitudes, comportamientos y conceptos relacionados con la sexualidad son construidos socialmente e incluyen creencias sobre las normas y tradiciones culturales, decisiones personales, influencia familiar y experiencias previas (Askun \& Ataca, 2007; Gelbal et al., 2008).

Así, las actitudes y comportamientos sexuales están determinados por tres niveles: cultural (normas sociales alrededor de la conducta sexual); interpersonal (transformación de los deseos sexuales en estrategias); y psicológico (diálogos sexuales con uno mismo y el manejo de la excitación) (Davidson et al., 2008). De este modo, las actitudes sexuales se configuran en el tiempo como un rasgo de la personalidad, resultado de la experiencia individual y la interacción con el entorno social, cultural y familiar (Borges et al., 2015) al que subyacen unos componentes cognitivos-emocional y comportamental.

En el componente cognitivo de la actitud sexual convergen dos constructos, el conservadurismo y el liberalismo. El conservadurismo se caracteriza por una visión estática y moralista de la sexualidad, asociada a la procreación. Por su parte, el liberalismo hace referencia a mantener una visión de la sexualidad fundamentada en el placer y asociada a cualquiera de las otras posibilidades: ternura, procreación, comunicación; en el liberalismo se mantiene una posición abierta ante el aborto, la educación sexual, las relaciones prematrimoniales, entre otras (Rodríguez \& Farré, 2004; Guerra \& Gouveia, 2007; Moraleda, 1994).

Respecto al componente comportamental, la mayoría de las investigaciones revisadas para este estudio demuestran el uso frecuente de dos conceptos como ejes centrales en su marco referencial, la erotofobia y la erotofilia, los cuales hacen referencia a la disposición a responder ante los estímulos sexuales a lo largo de una dimensión negativa-positiva de afecto y evaluación (Fisher et al., 1988; Castro et al., 2004; Hendrick et al., 2006; Zubeitdat et al., 2003; Rosales \& 
Moral, 2001). La erotofobia es una disposición aprendida que resulta de la exposición, durante la socialización, a la restricción y al castigo relacionados con el sexo, cuyas consecuencias implican la evitación de las respuestas de acercamiento a la sexualidad.

De esta manera, las personas que tienden hacia la erotofobia suelen responder con emociones negativas ante los estímulos sexuales, evaluándolos de forma negativa. Así mismo, pueden experimentar escasas respuestas al erotismo, dificultades para tener fantasías sexuales, para aprender o enseñar a otros acerca del sexo, para participar en el cuidado de la salud relacionada con el sexo y demostrar baja disposición a utilizar la anticoncepción (Fisher et al., 1988). Por el contrario, las personas que se aproximan hacia el polo de la erotofilia, responderán con emociones positivas ante la estimulación sexual, evaluándola de forma positiva y mostrando una mayor tendencia a la aproximación y búsqueda de los estímulos sexuales, relacionados con más experiencias sexuales y mayor número de parejas, con el aprendizaje de información sexual y con la posibilidad del uso de la anticoncepción, además con una mayor satisfacción sexual (Diéguez et al., 2004), con la edad y el sexo de las personas (Sales \& Flokman, 2003; Moser et al., 2007; Rodríguez \& Farré, 2004; Vargas-Trujillo et al., 2006).

Dada la complejidad de las secuelas que pueden existir tras la lesión por TCE, los posibles efectos sobre la sexualidad pueden estar directamente relacionados con los daños estructurales y funcionales sufridos, o ser secundarios al impacto de la medicación o a reacciones psicológicas y emocionales como la depresión o la ansiedad. De igual forma, la etiología y los efectos del funcionamiento sexual después de padecer un TCE no suelen ser muy conocidos, pues la literatura actual no permite evaluar la contribución de cada problema a la disfunción sexual presentada. Por ello, es importante profundizar en el estudio de las estrategias empleadas por los afectados y/o sus parejas, para sobrellevar el proceso y entender cómo perciben las consecuencias del TCE sobre su salud sexual.

En este sentido, el objetivo de este estudio fue identificar las actitudes sexuales en pacientes con trauma craneoencefálico y conocer las diferencias existentes de acuerdo con el nivel de severidad del TCE. 


\section{Método}

Se realizó un estudio descriptivo-comparativo que buscó especificar las propiedades, características y perfiles de personas, grupos, comunidades, procesos, objetos o cualquier otro tipo de fenómeno que se someta a un análisis. En este sentido, bajo la comparación de ciertas características de los datos, este tipo de estudio permite describir tendencias de un grupo o población (Hernández et al., 2014).

\section{Tabla 1}

Variables del estudio

\begin{tabular}{|c|c|c|}
\hline Variable & Definición conceptual & Definición operacional \\
\hline $\begin{array}{l}\text { V1: Severidad del trauma } \\
\text { craneoencefálico }\end{array}$ & $\begin{array}{l}\text { Se define teniendo en cuenta descriptores médicos } \\
\text { tales como duración de la pérdida de conciencia y } \\
\text { de la amnesia postraumática, la aparición de una } \\
\text { fractura de cráneo y la presencia de hematomas } \\
\text { intra o extracerebrales. Se mide a través de la Escala } \\
\text { de Coma de Glasgow, que incluye tres aspectos } \\
\text { fundamentales: apertura ocular, respuesta motora y } \\
\text { respuesta verbal; de allí se clasifica el trauma en grave } \\
\text { (8 o menos), moderado (9-13) leve (14-15) (MacNeill } \\
\& \text { Wedding, 2008). }\end{array}$ & $\begin{array}{l}\text { Es una variable cualitativa medida en escala } \\
\text { ordinal y variación discreta que se mide a través } \\
\text { de la Escala de Coma de Glasgow, la cual clasifica } \\
\text { el TCE en grave ( } 8 \text { o menos), moderado (9-13) } 0 \\
\text { leve (14-15). }\end{array}$ \\
\hline V2: Actitudes sexuales & $\begin{array}{l}\text { Son tendencias psicológicas que se expresan al } \\
\text { evaluar la sexualidad, que predisponen a evaluar } \\
\text { favorable o desfavorablemente los objetos y sujetos } \\
\text { que nos rodean en relación con la conducta sexual } \\
\text { (Bautista Hernández, 2008) }\end{array}$ & $\begin{array}{l}\text { Variable cualitativa medida en escala ordinal y } \\
\text { variación discreta, la cual se medirá a través de la } \\
\text { Escala de Actitudes Sexuales Ampliada de Fisher } \\
\text { y Hall adaptada por López, A. } 2005 \text {, la cual asume } \\
\text { los valores de conservadurismo, liberalismo como } \\
\text { parte del componente cognitivo de la actitud } \\
\text { y erotofilia y erotofobia como el componente } \\
\text { conductual. }\end{array}$ \\
\hline
\end{tabular}

\section{Participantes}

En el estudio participaron hombres y mujeres con antecedentes de trauma craneoencefálico. La selección de la muestra se realizó de manera no probabilística, atendiendo a los siguientes criterios de inclusión: a) encontrarse en un rango de edad entre los 18 y 49 años, b) haber pasado mínimo 6 meses después del trauma y presentar una adecuada comprensión verbal. Como criterios de exclusión: a) presencia de afasias, b) tener historia previa de discapacidad cognitiva, antecedentes de desarrollo, c) presencia de amnesia postraumática, d) presentar alteraciones sensitivas y/o motoras, e) presentar antecedentes neurológicos y/o psiquiátricos previos al trauma. 
De tal modo, la muestra quedó constituida por 126 participantes, de los cuales 91 son hombres y 35 mujeres, con un promedio de edad de 33 años, en su mayoría solteros, con antecedentes de trauma craneoencefálico superior a un año. Con relación a la severidad del trauma, participaron 39 hombres y 19 mujeres con TCE leve (46\%), 23 hombres y 13 mujeres con TCE moderado (28 \%) y 29 hombres y 3 mujeres con TCE severo (26\%). De igual forma, se encontró que, independientemente del nivel de severidad, la causa del TCE estuvo asociada a un accidente de tránsito, seguida por caídas; en este mismo sentido, prevaleció el TCE de tipo cerrado (Ver Tabla 2).

Tabla 2

Datos sociodemográficos y clínicos

\begin{tabular}{|c|c|c|c|c|c|c|}
\hline & Descripción & Leve & Moderado & Severo & Total & Porcentaje \\
\hline \multirow[t]{2}{*}{ Sexo } & Hombre & 39 & 23 & 29 & 91 & $72 \%$ \\
\hline & Mujer & 19 & 13 & 3 & 35 & $28 \%$ \\
\hline \multirow[t]{6}{*}{ Estado civil } & Soltero(a) & 20 & 14 & 17 & 51 & $40 \%$ \\
\hline & Separado(a) & 2 & 2 & 3 & 7 & $6 \%$ \\
\hline & Casado(a) & 13 & 10 & 8 & 31 & $25 \%$ \\
\hline & Viudo(a) & 1 & 0 & 0 & 1 & $0,8 \%$ \\
\hline & Divorciado(a) & 1 & 0 & 0 & 1 & $0,8 \%$ \\
\hline & Unión libre & 21 & 10 & 4 & 35 & $27 \%$ \\
\hline \multirow[t]{6}{*}{ Causa del trauma } & Accidente de tránsito & 50 & 23 & 28 & 101 & $80 \%$ \\
\hline & Caída & 3 & 10 & 2 & 15 & $12 \%$ \\
\hline & Arma de fuego & 1 & 1 & 1 & 3 & $2 \%$ \\
\hline & Arma corto punzante & 3 & 0 & 1 & 4 & $3 \%$ \\
\hline & MAP & 0 & 2 & 0 & 2 & $2 \%$ \\
\hline & Golpe contundente & 1 & 0 & 0 & 1 & $1 \%$ \\
\hline \multirow[t]{2}{*}{ Tipo de trauma } & Abierto & 15 & 8 & 10 & 33 & $26 \%$ \\
\hline & Cerrado & 42 & 28 & 23 & 93 & $74 \%$ \\
\hline
\end{tabular}

\section{Instrumentos}

Escala de coma de Glasgow (ECG): tiene como objetivo estandarizar la evaluación del nivel de consciencia en pacientes con traumatismo craneoencefálico, valorar la evolución neurológica de pacientes con este y determinar objetivamente la severidad de la disfunción cerebral, aparte de crear una comunicación confiable y certera entre profesionales de la salud. La ECG se compone de 3 subescalas que califican de manera individual 3 aspectos de la consciencia: la apertura ocular en un rango de 1 a 4 puntos, la respuesta verbal en rango de 1 a 5 puntos y la respuesta motora que va de 1 a 6 puntos; el puntaje total se otorga con base en la mejor respuesta obtenida en cada uno de los rubros (Munana-Rodríguez \& Ramírez-Elías, 2014). En general, se puede clasificar como TCE severo, de 0 a 8 puntos; TCE moderado, de 9 a 13 puntos; y TCE leve, de 14 a 15 puntos. 
Escala de Actitudes hacia la Sexualidad Ampliada (Fisher \& Hall, 1988, adaptada por Diéguez et al., 2005): este instrumento consta de 28 ítems (luego de ser adaptado por Diéguez, et al., 2005), y toma como base la Escala de Actitudes hacia la Sexualidad Ampliada (ATSS) de Fisher y Hall (1988), de 14 ítems. La Escala está construida en el marco de los conceptos de actitud positiva o negativa (conservadora o liberal) hacia diferentes aspectos centrales de la sexualidad y corresponde con la medición de las actitudes mediante el rendimiento en tareas objetivas. Cada una de las afirmaciones pertenece a uno de los factores en los que fueron agrupados los 28 ítems. El factor 1 incluye aquellos que evalúan la responsabilidad, libertad y opinión que merece la juventud; el factor 2 hace referencia a la pornografía y la prostitución; el factor 3 incluye ítems relativos a la masturbación, las fantasías y la educación sexual; el factor 4 incluye ítems enfocados hacia los lugares nudistas, las caricias y el coito fuera del matrimonio; el factor 5 se refiere a la conducta homosexual, las enfermedades de trasmisión sexual y la libertad de cada persona ante las conductas sexuales; finalmente, el factor 6 incluye asuntos relativos a la evolución sexual, el aborto, el coito con afecto y la educación sexual en la familia y en la escuela. La consistencia interna de la escala es de ,84. Su validez pone de manifiesto la multidimensionalidad de la escala, una vez cuenta con validez de constructo, obtenida mediante un análisis factorial por el método de componentes principales.

Encuesta Actitudes hacia la Sexualidad (Arango et al., 2014): para determinar cómo los pacientes con trauma craneoencefálico (TCE) y sus parejas perciben su sexualidad tras el incidente y el abordaje que los profesionales le dan, un grupo de investigadores profesionales de la Universidad de Deusto, en España, desarrollaron dos encuestas: una para el paciente con TCE y otra para la pareja. Primero, se hizo una revisión exhaustiva de la literatura para identificar información acerca de la percepción de la sexualidad tras el TCE, la relación que los pacientes mantienen con la pareja y con el equipo de rehabilitación con respecto al tema, y el asesoramiento y la rehabilitación recibida para superar o mejorar su vida sexual, todo ello para crear las preguntas. Luego, las encuestas se enviaron a un grupo de expertos (5 expertos en Latinoamérica y Estados Unidos), para evaluar que las preguntas estuvieran adaptadas a la cultura y al contexto lingüístico del país, así como su aplicabilidad y valor. Varios de los comentarios de los expertos fueron incorporados y las encuestas quedaron compuestas de 59 preguntas (versión de pacientes) y 50 preguntas (versión de parejas). De esta manera, el instrumento contó con validación por jueces. Las preguntas fueron agrupadas en información sociodemográfica, funcionamiento sexual, relación con la pareja (sólo aplicable a personas que tuvieran pareja), y con el equipo de rehabilitación, asesoramiento sexual y rehabilitación sexual.

Para este estudio se tomaron las preguntas relacionadas con la importancia de la sexualidad, cambios en la sexualidad tras el TCE, aspectos físicos alterados, deseo sexual, cambios para alcanzar el orgasmo, frecuencia de la masturbación después del TCE, presencia de pareja actual y 
frecuencia de las relaciones sexuales posterior al trauma. Estas preguntas son pertenecientes a las categorías sexualidad en general y relación de pareja, dada su afinidad con los ítems de la Escala de Actitudes hacia la Sexualidad Ampliada de Fisher y Hall (ATSS) (Diéguez et al., 2005).

\section{Resultados}

Para el análisis de los resultados se emplearon medidas de tendencia central y una prueba de hipótesis chi-cuadrada. Como se observa en la Tabla 2, el $46 \%$ reportó haber sufrido un TCE leve, de tipo cerrado, y a causa de un accidente de tránsito; por su parte, el $28 \%$ reportó un TCE moderado; y el 26 \% restante un TCE severo -en su mayoría hombres (29, respecto a 3 mujeres)-.

En la Tabla 3 se presenta la valoración obtenida en cada componente de la Escala ATSS, destacándose la importancia que otorgaron los participantes a los cambios en la sexualidad tras el TCE, así como aspectos cognitivos (atención, memoria, lenguaje) y estados emocionales alterados (ansiedad, depresión, baja autoestima), deseo sexual, fantasías sexuales, cambio de roles, frecuencia de las relaciones sexuales posterior al trauma y cambios en la masturbación; se relacionó cada uno de estos ítems con los componentes de la actitud (cognitivo, afectivo y comportamental).

En cuanto al componente cognitivo, no hubo diferencias significativas con respecto al nivel de severidad del trauma. Por ejemplo, la pregunta relacionada con la importancia de la sexualidad evidenció que el $85 \%$ de los participantes, independiente del grado de severidad del trauma, la consideran importante. El 61 \% de los pacientes reportó una disminución del deseo sexual, encontrando diferencia respecto al TCE leve (Ver Tabla 3). Por su parte, las preguntas relacionadas con cambios emocionales (ansiedad, depresión y baja autoestima) y fantasías sexuales no revelaron diferencias significativas respecto al TCE y el nivel de severidad. En cuanto a las preguntas relacionadas con el componente conductual, se encontró que el $51 \%$ de los participantes ha experimentado cambios en la sexualidad, independiente del nivel de severidad.

La mayoría de los participantes reporta algún tipo de cambio en su sexualidad y en la valoración de esta tanto a nivel cognitivo, como emocional o comportamental, una vez el componente sexual constituye un elemento fundamental en su relación de pareja; como se mencionaba anteriormente, el $52 \%$ de ellos tenía algún vínculo afectivo o sexual vigente en el momento de la 
investigación (Ver Tabla 2). También fue posible observar cómo algunas de las respuestas fueron sesgadas por aspectos como pudor, desconocimiento del tema -por ejemplo en cuanto al significado de fantasías sexuales- y la nula posibilidad de realimentar al participante al momento de responder a la encuesta, dado el alto grado de confidencialidad con el que fueron manejados los instrumentos.

Tabla 3

Resultados de la Encuesta de Actitudes hacia la Sexualidad respecto al nivel de severidad del TCE.

\begin{tabular}{|c|c|c|c|c|c|c|c|}
\hline \multirow{2}{*}{$\begin{array}{l}\text { Componente de } \\
\text { la actitud }\end{array}$} & \multirow{2}{*}{ Ítem } & \multirow{2}{*}{ Descripción } & \multicolumn{3}{|c|}{ Severidad TCE } & \multirow{2}{*}{ Total } & \multirow{2}{*}{$\%$} \\
\hline & & & Leve & Moderado & Severo & & \\
\hline \multirow{5}{*}{ Cognitivo } & \multirow{3}{*}{ Importancia sexualidad } & Muy importante & 25 & 16 & 16 & 57 & 45 \\
\hline & & Importante & 25 & 14 & 11 & 50 & 40 \\
\hline & & Poco importante & 8 & 6 & 5 & 19 & 15 \\
\hline & \multirow{2}{*}{$\begin{array}{l}\text { Cambios a nivel cognitivo } \\
\text { (atención, memoria, lenguaje) }\end{array}$} & $\mathrm{Si}$ & 18 & 6 & 9 & 33 & 26 \\
\hline & & No & 40 & 30 & 23 & 93 & 74 \\
\hline \multirow{6}{*}{ Emocional } & \multirow{2}{*}{$\begin{array}{l}\text { Cambios a nivel emocional } \\
\text { (depresión, ansiedad, baja } \\
\text { autoestima) }\end{array}$} & $\mathrm{Si}$ & 16 & 12 & 7 & 35 & 28 \\
\hline & & No & 42 & 24 & 25 & 91 & 72 \\
\hline & \multirow{2}{*}{ Cambios en el deseo sexual } & Igual & 18 & 19 & 12 & 49 & 39 \\
\hline & & Ha cambiado & 40 & 17 & 20 & 77 & 61 \\
\hline & \multirow{2}{*}{ Fantasías sexuales } & Igual & 42 & 28 & 20 & 90 & 71 \\
\hline & & Ha cambiado & 16 & 8 & 12 & 36 & 29 \\
\hline \multirow{10}{*}{ Conductual } & \multirow{2}{*}{ Cambio sexualidad } & $\mathrm{Si}$ & 30 & 15 & 19 & 64 & 51 \\
\hline & & No & 28 & 21 & 13 & 62 & 49 \\
\hline & \multirow{2}{*}{$\begin{array}{l}\text { Cambios en roles } \\
\text { (dependencia) }\end{array}$} & Si & 10 & 6 & 1 & 17 & 13 \\
\hline & & No & 48 & 30 & 31 & 109 & 87 \\
\hline & \multirow{4}{*}{$\begin{array}{l}\text { Frecuencia de las relaciones } \\
\text { sexuales }\end{array}$} & Ha aumentado & 8 & 3 & 6 & 17 & 13 \\
\hline & & Ha disminuido & 15 & 8 & 10 & 33 & 26 \\
\hline & & Se mantienen igual & 9 & 10 & 7 & 26 & 21 \\
\hline & & No aplica & 26 & 15 & 9 & 50 & 40 \\
\hline & \multirow{2}{*}{ Cambios en la masturbación } & No & 31 & 32 & 21 & 84 & 67 \\
\hline & & $\overline{\mathrm{Si}}$ & 27 & 4 & 11 & 42 & 33 \\
\hline
\end{tabular}


Respecto a la Escala de Actitudes hacia la Sexualidad Ampliada ATSS, todos los participantes, independientemente del nivel de severidad del trauma craneoencefálico, obtuvieron puntuaciones $\geq 80$ (Ver Tabla 4) lo cual guarda relación con lo presentado respecto de los cambios reportados por los pacientes en cuanto al deseo sexual, la frecuencia de las relaciones sexuales, la importancia y el cambio en la sexualidad.

Tabla 4

Resultados de la ATSS.

\begin{tabular}{|c|c|c|c|c|}
\hline & Severidad TCE & Estadístico & & Error típico \\
\hline \multirow{9}{*}{ Total de las encuestas } & \multirow{3}{*}{ Leve } & Media & 80,3 & 1,351 \\
\hline & & Mediana & 79 & \\
\hline & & DT & 10,3 & \\
\hline & \multirow{3}{*}{ Moderado } & Media & 80,9 & 1,389 \\
\hline & & Mediana & 80 & \\
\hline & & DT & 8,3 & \\
\hline & \multirow{3}{*}{ Severo } & Media & 79,6 & 1,581 \\
\hline & & Mediana & 79 & \\
\hline & & DT & 8,9 & \\
\hline
\end{tabular}

Contrario a lo anterior, no se encontraron diferencias significativas en cuanto a los factores o categorías mediante las cuales los autores de la ATSS agruparon los ítems y el nivel de severidad del TCE, tal como se observa en la Tabla 5, la cual presenta la relación entre cada uno de los factores de la ATSS y el nivel de severidad del TCE, y evidencia que, salvo en el factor 2, se encuentra una relación entre la valoración que hacen las personas sobre aspectos como la pornografía y la prostitución y el TCE leve, así mismo, en el factor 5, concerniente a la conducta homosexual, las enfermedades de transmisión sexual y la libertad de las personas ante la conducta homosexual, y los tres niveles de severidad del TCE (leve, moderado y severo). 
Tabla 5

Relación entre factores de la ATSS y el nivel de severidad del TCE.

\begin{tabular}{|c|c|c|c|c|c|}
\hline \multirow{2}{*}{ Factores } & \multirow{2}{*}{ Severidad del TCE } & \multicolumn{3}{|c|}{ Estadístico } & \multirow{2}{*}{$\begin{array}{l}\text { Error } \\
\text { tipico }\end{array}$} \\
\hline & & Media & Mediana & DT & \\
\hline \multirow{3}{*}{$\begin{array}{l}\text { Factor } 1 \text {. } \\
\text { Responsabilidad, libertad } \\
\text { y opinión que merece } \\
\text { la juventud }\end{array}$} & Leve & 18,06 & 18,5 & 4,84 & 0,636 \\
\hline & Moderado & 19 & 20 & 4,34 & 0,724 \\
\hline & Severo & 19,5 & 20 & 4,6 & 0,813 \\
\hline \multirow{3}{*}{$\begin{array}{l}\text { Factor } 2 . \\
\text { Pornografía y } \\
\text { prostitución }\end{array}$} & Leve & 6,98 & 7 & 2,98 & 0,391 \\
\hline & Moderado & 7,83 & 8,5 & 2,81 & 0,468 \\
\hline & Severo & 8,46 & 8 & 2,51 & 0,444 \\
\hline \multirow{3}{*}{$\begin{array}{l}\text { Factor } 3 . \\
\text { Masturbación, } \\
\text { fantasías y } \\
\text { educación sexual }\end{array}$} & Leve & 16,05 & 17 & 3,26 & 0,428 \\
\hline & Moderado & 16,33 & 16 & 3,69 & 0,615 \\
\hline & Severo & 14,9 & 15 & 3,17 & 0,561 \\
\hline \multirow{3}{*}{$\begin{array}{l}\text { Factor } 4 . \\
\text { Lugares nudistas, } \\
\text { caricias y } \\
\text { coito fuera } \\
\text { del matrimonio. }\end{array}$} & Leve & 14,15 & 14 & 3,87 & 0,509 \\
\hline & Moderado & 13,52 & 13 & 2,89 & 0,482 \\
\hline & Severo & 12,84 & 12 & 3,17 & 0,561 \\
\hline \multirow{3}{*}{$\begin{array}{l}\text { Factor } 5 . \\
\text { Conducta homosexual, } \\
\text { enfermedades } \\
\text { de trasmisión sexual }\end{array}$} & Leve & 8,96 & 9 & 2,15 & 0,282 \\
\hline & Moderado & 8,94 & 9 & 2,32 & 0,388 \\
\hline & Severo & 8,93 & 9 & 1,86 & 0,329 \\
\hline \multirow{3}{*}{$\begin{array}{l}\text { Factor } 6 . \\
\text { Evolución sexual, aborto, } \\
\text { coito con afecto y } \\
\text { educación sexual }\end{array}$} & Leve & 16,12 & 15,5 & 3,18 & 0,418 \\
\hline & Moderado & 15,22 & 14 & 2,77 & 0,463 \\
\hline & Severo & 14,93 & 15 & 3,24 & 0,573 \\
\hline
\end{tabular}

En las medidas de comparación entre las actitudes sexuales de los instrumentos utilizados y la severidad del TCE, tal como se evidencia en la Tabla 6, se encontraron las 6 correlaciones siguientes: 1) Factor 1 y eyaculación después del TCE (pregunta 23) respecto al TCE moderado, indicando ajuste entre las respuestas dadas por las personas con esta condición frente a temas como masturbación, eyaculación, fantasías y educación sexual; 2) Factor 6 y eyaculación después del TCE (pregunta 23) respecto al TCE leve, evidenciando una correlación entre la evolución sexual y el coito con afecto frente a la eyaculación después del TCE leve; 3) Factor 6 y cambios para alcanzar el orgasmo (pregunta 27) respecto al TCE moderado, evidenciando la relación entre la severidad moderada del TCE y la capacidad para alcanzar el orgasmo, la evolución y la educación sexual; 4) Factor 3 y frecuencia de masturbación después del TCE (pregunta 29) respecto al TCE leve, indicando un alto nivel de significancia entre las preguntas y las respuestas dadas por los participantes respecto a la frecuencia de masturbación después del TCE leve y la actitud hacia esta conducta y las fantasías sexuales; 5) Factor 5 y frecuencia de masturbación después del TCE (pregunta 29) respecto al TCE leve, evidenciando la relación entre las preguntas que abordan la frecuencia de masturbación después del TCE y la libertad ante las conductas sexuales evaluadas 
en la ATSS; 6) Factor 2 y fantasías sexuales (pregunta 30) con relación al TCE leve, encontrándose correlación entre la evaluación que se hace sobre temas como la pornografía y la capacidad para tener fantasías sexuales.

Tabla 6

Relación entre Factores ATSS y preguntas de la Encuesta de Actitudes hacia la sexualidad respecto al nivel de severidad del TCE.

\begin{tabular}{|c|c|c|c|c|}
\hline \multirow{2}{*}{$\begin{array}{l}\text { Factores vs } \\
\text { preguntas }\end{array}$} & \multirow{2}{*}{$\begin{array}{c}\text { Nivel de Severidad del } \\
\text { TCE }\end{array}$} & \multicolumn{3}{|c|}{ Chi-cuadrado de Pearson } \\
\hline & & Valor & gl & Sig. asintótica (bilateral) \\
\hline \multirow{3}{*}{$\begin{array}{l}\text { Factor } 3 \text { y eyaculación } \\
\text { después del TCE } \\
\text { (pregunta 23) }\end{array}$} & Leve & 8,019 & 12 & 0,78 \\
\hline & Moderado & 26,000 & 12 & 0,01 \\
\hline & Severo & 10,142 & 9 & 0,33 \\
\hline \multirow{3}{*}{$\begin{array}{l}\text { Factor } 6 \text { y eyaculación } \\
\text { después del TCE } \\
\text { (pregunta 23) }\end{array}$} & Leve & 30,160 & 12 & 0 \\
\hline & Moderado & 11,917 & 9 & 0,21 \\
\hline & Severo & 5,954 & 9 & 0,74 \\
\hline \multirow{3}{*}{$\begin{array}{l}\text { Factor } 6 \text { y cambios } \\
\text { para alcanzar el } \\
\text { orgasmo (pregunta 27) }\end{array}$} & Leve & 14,917 & 13 & 0,31 \\
\hline & Moderado & 21,656 & 10 & 0,01 \\
\hline & Severo & 7,039 & 11 & 0,79 \\
\hline \multirow{3}{*}{$\begin{array}{l}\text { Factor } 3 \text { y frecuencia } \\
\text { de masturbación } \\
\text { después de TCE } \\
\text { (pregunta 29) }\end{array}$} & Leve & 76,136 & 36 & 0 \\
\hline & Moderado & 16,531 & 12 & 0,16 \\
\hline & Severo & 13,537 & 11 & 0,26 \\
\hline \multirow{3}{*}{$\begin{array}{l}\text { Factor } 5 \text { y frecuencia } \\
\text { de masturbación } \\
\text { después de TCE } \\
\text { (pregunta 29) }\end{array}$} & Leve & 46,636 & 30 & 0,02 \\
\hline & Moderado & 7,042 & 9 & 0,63 \\
\hline & Severo & 4,124 & 7 & 0,76 \\
\hline \multirow{3}{*}{$\begin{array}{l}\text { Factor } 2 \text { y fantasías } \\
\text { sexuales } \\
\text { (pregunta 30) }\end{array}$} & Leve & 20,312 & 10 & 0,02 \\
\hline & Moderado & 7,843 & 9 & 0,55 \\
\hline & Severo & 8,960 & 8 & 0,34 \\
\hline
\end{tabular}

\section{Discusión}

Los resultados obtenidos durante la investigación evidencian que los participantes percibieron cambios en su sexualidad tras sufrir un trauma craneoencefálico, los cuales estuvieron relacionados con aspectos como la disminución en la frecuencia de las relaciones sexuales, la capacidad para tener fantasías sexuales y cambios para lograr el orgasmo; sin embargo, manifestaron que consideran la sexualidad como un aspecto importante en su vida, lo que indica una tendencia homogénea de los participantes a exhibir una actitud sexual liberalista, independiente del nivel de severidad del trauma. Lo anterior es congruente con lo planteado por Ariza-González et al. (2004), quienes reportan los cambios emocionales que tienen lugar como resultado del trauma cerebral e incluyen la exageración o desaparición de la experiencia y la respuesta emocional. 
También fue posible evidenciar la disociación entre el componente cognitivo (liberalismo) y el componente conductual (erotofilia) de la actitud sexual, una vez que los participantes, independientemente del nivel de severidad del trauma, expresaron una tendencia a la valoración positiva de la sexualidad, pero manifestaron disminución en las conductas de aproximación y búsqueda de estímulos sexuales, tal como lo reflejan los resultados de las preguntas relacionadas con la disminución en la frecuencia de las relaciones y el deseo sexual; lo que permite inferir que aunque las personas evaluadas tienen una valoración positiva de la sexualidad, este asunto no se relaciona con la experiencia de vida sexual actual tras el TCE.

La interpretación anterior es congruente con los estudios realizados por Arango-Lasprilla et al. (2007), quienes afirman que la lesión cerebral puede provocar una afectación directa o indirecta en aspectos como la sexualidad y la función sexual. En igual sentido, Creuter et al. (2009) manifiestan que los daños en el lóbulo frontal desencadenan cambios en el sistema de activación sexual, tales como disminución del deseo y de la excitación genital.

Respecto al sexo, se contó con mayor participación de hombres con antecedentes de TCE severo, siendo esto coherente con lo reportado en la literatura, la cual ofrece indicios de que existe un mejor procesamiento emocional en las mujeres y un control más eficiente de las conductas de riesgo y conductas socialmente inadecuadas, por ejemplo: actividades que impliquen altas velocidades o deportes extremos que desencadenen en posibles accidentes de tránsito y caídas. Así mismo, es importante mencionar que, aunque la muestra incluyó pacientes con TCE de todos los niveles de severidad, el número de pacientes con TCE severo fue menor respecto a los demás niveles, considerando que las personas con traumatismos graves tienen un mayor riesgo de muerte y menor posibilidad de recuperación, lo cual pudo afectar los resultados de la investigación de no haberse considerado dentro de los criterios de inclusión para la muestra.

De esta manera, los resultados permiten concluir que las actitudes sexuales desempeñan un papel destacado en la sexualidad del ser humano y, de acuerdo con lo mencionado por Ortega et al. (2005), aquellos individuos que manifiestan actitudes sexuales positivas suelen gozar de una mayor satisfacción sexual, e incluso, suelen demostrar más habilidades en la prevención de enfermedades de transmisión sexual, tal como lo revelan los resultados, pues los participantes se caracterizaron por manifestar una actitud sexual liberalista o evaluaron positivamente la sexualidad, independientemente del nivel de severidad del trauma (leve, moderado y severo), lo que significa una adecuada capacidad para el aprendizaje de información sexual, la posibilidad del uso de anticoncepción, la tendencia a aprender o enseñar a otros acerca del sexo y la participación en el cuidado de la salud en relación con el sexo y el comportamiento sexual durante el embarazo y el posparto. 
Ahora bien, es importante considerar que los pacientes no recibieron realimentación respecto a las respuestas dadas tanto en la encuesta como en la Escala de Actitudes hacia la Sexualidad Ampliada en el momento de la aplicación, dado el nivel de confidencialidad con que se acordó la evaluación, lo que pudo influir en la emisión de respuestas convenientes y probablemente escasas de realidad, permitiendo evidenciar también la pérdida de la conciencia de los propios déficit y falta de habilidad para percibir los cambios en las funciones cognitivas y en la conducta que presentan los pacientes con TCE.

Dentro de las conclusiones de la investigación, puede mencionarse que la sexualidad constituye un elemento fundamental en la configuración de la personalidad y determina el comportamiento basado en el género, lo cual se evidenció en la tendencia clara de la muestra hacia la valoración positiva de la sexualidad, pese a que esto no guardara relación con su situación sexual actual, pues podría pensarse que ante una valoración de este nivel existiera una mayor tendencia o aproximación hacia conductas sexuales.

Para terminar, es importante robustecer la investigación sobre sexualidad y TCE con el fin de establecer nuevas rutas para la rehabilitación de los pacientes, sobre todo si se considera el gran número de personas afectadas que deja el conflicto armado en Colombia y demás situaciones de violencia y riesgo, así como el reto que esto impone a la comunidad de neuropsicólogos, en la medida que se requieren programas de intervención más sensibles, pertinentes y eficientes que involucren objetivos encaminados a explorar y mejorar la comprensión de los inevitables cambios en la sexualidad que conlleva un TCE, independientemente de su nivel de severidad.

\section{Conflicto de intereses}

Las autoras declaran la inexistencia de conflicto de interés con institución o asociación comercial de cualquier índole. 


\section{Referencias}

Albadan-Amaya, M., Sánchez-Lozano, M., \& Vergara-Torres, G. (2013). Respuesta sexual en una muestra de hombres que han sufrido un trauma craneoencefálico moderado-severo. $R e$ vista Virtual Psicoespacios, 7(11), 216-228. http://bibliotecadigital.iue.edu.co/jspui/bitst ream/20.500.12717/2598/1/226-Texto\%20del\%20art\%c3\%adculo-4630-1-10-20200910. pdf

Arango-Lasprilla, J. C., Rosenthal, M., De Luca, J., Cifu, D. X., Hanks, R., \& Komaroff, E. (2007). Functional Outcomes from Inpatient Rehabilitation after Traumatic Brain Injury: How Do Hispanics Fare. Archives of Physical Medicine and Rehabilitation, 88(1), 11-8. https://doi.org/10.1016/j.apmr.2006.10.029

Ariza-González, M., Pueyo-Benito, R., \& Serra-Grabulosa, J. M. (2004). Secuelas neuropsicológicas de los traumatismos craneoencefálicos. Anales de Psicología, 20(2), 303-316. https://www.um.es/analesps/v20/v20_2/10-20_2.pdf

Askun, D., \& Ataca, B. (2007). Sexuality Related Attitudes and Behaviors of Turkish University Students. Archives of Sexual Behavior, 36(5), 741-752. https://doi.org/10.1007/s10508007-9186-z

Barcena-Orbe, A., Rodríguez-Arias, C.A., Rivero-Martín, B., Cañizal-García, J., Mestre-Moreiro, C., Calvo-Pérez, J. C., Molina-Foncea, A. F., \& Casado-Gómez, J. (2006). Revisión del traumatismo craneoencefálico. Revista Neurocirugía, 17(6) 495-518. https://doi. org/10.1016/S1130-1473(06)70314-1

Bautista-Hernández, L. (2008). Conocimientos, actitudes y opiniones de las y los universitarios oaxaqueños respecto a su sexualidad y la importancia de la educación sexual en el nivel de educación superior. Centro Regional de Investigación en Psicología, 2(1), 71-84. http:// www.imbiomed.com.mx/1/1/articulos.php?method=showDetail\&id_articulo=51596\&id_ seccion $=3247 \&$ id_ejemplar $=5224 \&$ id_revista $=170$

Bigler, E. (2001). The Lesion(s) in Traumatic Brain Injury: Implications for Clinical Neuropsychology. Archives of Clinical Neuropsychology, 16(2), 95-131. https://doi.org/10.1016/ S0887-6177(00)00095-0

Borges, I., Costa, V., \& Quintas, M. (2015). The Family Organizational Culture as Predictors of Sexual Attitudes and Behaviors in Adolescents. Journal of Nursing Reference, 4(6), 17-24. 
Castaño-Monsalve, B., Bernabeu-Guitart, M., López, R., Bulbena-Vilasar, A., \& Ignacio-Quemada, J. (2012). Perfil psicopatológico de pacientes con traumatismo craneoencefálico evaluados mediante el Inventario Neuropsiquiátrico. Revista de Psiquiatría y Salud Mental, 5(3), 160-166. https://doi.org/10.1016/j.rpsm.2012.02.004

Castro, M. E., Sueiro, E., López, A., \& Cortegoso, M. (2004). Conocimientos y actitudes hacia la sexualidad de agresores sexuales antes y después de un programa educativo. (Estudio piloto). Psicosoma, (69-70), 78-94. http://www.editorialmedica.com/download.php?idart=177

Creuter, M., Dahllof, A., Gudjonsson, G., Sullivan, M., \& Siosteen, A. (2009). El ajuste sexual y sus predictores después de una lesión cerebral traumática. Gotemburgo

Fontaine, K., Moore, N., Earle, J., \& Davis, R. (2008). Sexual Attitudes and Behavior at Four Universities: Do Region, Race, and/or Religion Matter? Adolescence, 43(170), 189-220. https://pubmed.ncbi.nlm.nih.gov/18689097/

Diéguez, J., Díaz, M. C., Sueiro, E., \& Chas, M. D. (2004). Primeras fuentes de información, conocimientos, actitudes y prácticas sexuales. Su interrelación. Psicosoma, (69-70), 95-114. https://dialnet.unirioja.es/servlet/articulo?codigo $=1195077$

Diéguez, J., López, A., Sueiro, E., \& López, F. (2005). Propiedades psicométricas de la Escala de Actitudes hacia la Sexualidad (ATSS) ampliada. Revista Iberoamericana de Psicosomática, (74), 46-56. https://dialnet.unirioja.es/servlet/articulo? codigo=1368867

Fisher, W., White, L., Byrne, D., \& Kelley, K. (1988). Erotophobia-erotophilia as a Dimension of Personality. Journal of Sex Research, 25(1), 123-151. https://doi. org/10.1080/00224498809551448

Fisher, T., \& Hall, R. (1988). A Scale for the Comparison of the Sexual Attitudes of Adolescents and Their Parents. The Journal of Sex Research, 24(1), 90-100. https://doi. org/10.1080/00224498809551400

Fontaine, A., Azouvi, P., Remy, P., Bussel, B., \& Samson, Y. (1999). Functional Anatomy of Neuropsychological Deficits after Severe Traumatic Brain Injury. Neurology, 53(9), 19631968. https://doi.org/10.1212/WNL.53.9.1963

Gálvez-González, M., Del Águila-Hidalgo, B., Campos-Gómez, M., Calle-Ruíz, M. y SaraibaHerrera, J., (2009). Perfil del paciente con traumatismo craneoencefálico grave en la provincia de Málaga. Tempus Vitalis, 9(1), 20-29. https://docplayer.es/18513614-Perfil-delpaciente-con-traumatismo-craneoencefalico-grave-en-la-provincia-de-malaga.html 
García-Molina, A., Bernabéu, M., \& Roig-Rovira, T. (2010). Traumatismo craneoencefálico y vida cotidiana: el papel de las funciones ejecutivas. Psicothema, 22(3), 430-435. http:// www.psicothema.com/pdf/3748.pdf

García-Molina, A., Roig-Rovira, T., Yuguero, M., Enseñat-Cantallops, A., Sánchez-Carrión, R., \& Bernabéu, M. (2008). La integración en la comunidad como medida de resultado de la neurorrehabilitación en el traumatismo craneoencefálico. Rehabilitación, 42(2), 67-72. https://www.redalyc.org/pdf/727/72714400012.pdf

Gelbal, S., Duyan, V., \& Ozturk, A. (2008). Gender Differences in Sexual Information Sources, and Sexual Attitudes and Behaviors of University Students in Turkey. Social Behavior and personality, 36(8), 1035-1052. https:/www.sbp-journal.com/index.php/sbp/article/ view/1778

González, B. (Coord.). (2012). Daño cerebral adquirido. Evaluación, diagnóstico y rehabilitación. Editorial síntesis.

Guerra, V., \& Gouveia, V. (2007). Liberalismo/conservadurismo sexual: Proposta de uma medida multi-fatorial. Psicologia: Reflexão e Crítica, 20(1), 43-53. https://www.scielo.br/j/prc/a/ L8sk5nwPwqgwsncgTmhWZsN/abstract/?lang=en

Guzmán, F. (2008). Fisiopatología del trauma craneoencefálico. Colombia Médica, 39(3), 78-84. http://www.redalyc.org/articulo.oa?id=28309611

Hendrick, C., Hendrick, S., \& Reich, D. (2006). The Brief Sexual Attitudes Scale. The Journal of Sex Research, 43(1), 76-86. https://doi.org/10.1080/00224490609552301

Hernández, R. Fernández, C., \& Baptista, P. (2014). Metodología de la investigación (6 ${ }^{a}$. ed.). McGraw-Hill Interamericana.

MacNeill, A., \& Wedding, D. (Eds.). (2008). The Neuropsychology Handbook. Springer Publishing Company.

Mendoza-Álvarez, L. A., García-Núñez, L. M., \& Arroyo-Mayorga, G. (2003). Epidemiología del trauma craneoencefálico evaluado por tomografía axial computada. Revista de Sanidad Militar, 57(5), 299-302. https://www.medigraphic.com/pdfs/sanmil/sm-2003/sm035c. pdf

Moraleda, M. (1994). Estudio comparativo de las actitudes y conducta sexual de los adolescentes y jóvenes españoles 1977-1992. Revista Complutense de Educación, 5(2), 199-207. https://revistas.ucm.es/index.php/RCED/article/view/RCED9494220199A 
Moser, A., Reggiani, C., \& Urbanetz, A. (2007). Comportamento sexual de risco entre estudantes universitárias dos cursos de ciências da saúde. Revista da Associação Médica Brasileira, 53(2), 116-121. http://dx.doi.org/10.1590/S0104-42302007000200014

Munana-Rodríguez, J. E., \& Ramírez-Elías, A. (2014). Escala de Coma de Glasgow: origen, análisis y uso apropiado. Enfermería Universitaria, 11(1), 24-35. https://doi.org/10.1016/ S1665-7063(14)72661-2

Organización Mundial de la Salud (OMS). (2015). Informe sobre la situación mundial de la seguridad vial 2015. https://www.who.int/violence_injury_prevention/road_safety_status/2015/es/

Ortega, V., Ojeda, P., Sutil, F., \& Sierra, J. (2005). Culpabilidad sexual en adolescentes: Estudio de algunos factores relacionados. Anales de Psicología, 21(2), 268-275. https://revistas. um.es/analesps/article/view/26861/26061

Rodríguez, S., \& Farré J. M. (2004). Actitudes sexuales: estudio piloto intergeneracional en un medio urbano. Psicosoma, (69-70), 61-77. http://www.sidastudi.org/es/registro/2c9391e4 $1 \mathrm{fb} 402 \mathrm{cc} 011 \mathrm{fb} 4462 \mathrm{e} 07493 \mathrm{a}$

Rodríguez, J., Mederos, A., Cisneros, M., \& Estrada, M. (2000). Trauma craneal leve. Revista Cubana de Medicina Militar, 29(1), 49-51. http://scielo.sld.cu/pdf/mil/v29n1/mil08100. pdf

Rosales, F., \& Moral, J. (2001). Sexo, autoeficacia y actitud hacia conductas sexuales de riesgo en población adolescente. Medicine Psicosomatic, (16), 207-220. https://dialnet.unirioja. es/servlet/articulo?codigo $=2282637$

Sales, B., \& Folkman, S. (Eds.). (2003). Ethics in Research with Human Participants. Blackwell Publishing.

Silver, J., McAllister, T., \& Yudofsky, S. (2005). Textbook of Traumatic Brain Injury (1rst ed.). American Psychiatric Publishing.

Spencer, L., Potterton, R., Allen, K., Musiat, P., \& Schmidt, U. (2019). Internet-Based Interventions for Carers of Individuals With Psychiatric Disorders, Neurological Disorders, or Brain Injuries: Systematic Review. Journal of Medical Internet Research, 21(7), e-10876. doi: $10.2196 / 10876$

Stein, D. M., Feather, C. B., \& Napolitano, L. M. (2017). Traumatic Brain Injury Advances. Critical Care Clinics, 33(1), 1-13. https://doi.org/10.1016/j.ccc.2016.08.008 
Varela, A., Pardo, G., Medrano, R., \& Rivero, C. (2005). Anormalidades tomográficas en el trauma craneoencefálico leve. Revista Mexicana de Neurociencia, 6(2), 120-123. http://www. sld.cu/galerias/pdf/sitios/neuroc/alteraciones_tomograficas_en_tce_leve.pdf

Vargas-Trujillo, E., Barrera, F., Burgos, M. C., \& Daza, B. C. (2006). La intención de los jóvenes de tener relaciones sexuales en la adolescencia: el papel de la televisión, la relación con los padres y las cogniciones. Universitas Psicológica, 5(1), 69-84. https://www.redalyc. org/pdf/647/64750106.pdf 